\title{
Antioxidant treatment of hindlimb-unloaded mouse counteracts fiber type transition but not atrophy of disused muscles
}

\author{
Jean-François Desaphy ${ }^{a}$, Sabata Pierno ${ }^{a}$, Antonella Liantonio ${ }^{a}$, Viviana Giannuzzi ${ }^{a}$, \\ Claudio Digennaro ${ }^{a}$, Maria Maddalena Dinardo ${ }^{a}$, Giulia M. Camerino ${ }^{a}$, Patrizia Ricciuti ${ }^{b}$, \\ Lorenza Brocca $^{c}$, Maria Antonietta Pellegrino ${ }^{c}$, Roberto Bottinelli ${ }^{c}$, Diana Conte Camerino ${ }^{\mathrm{a}, *}$ \\ a Section of Pharmacology, Department of Pharmacobiology, Faculty of Pharmacy, University of Bari, Via Orabona 4 - campus, 70125 Bari, Italy \\ ${ }^{\mathrm{b}}$ Department of Agro-Forestry and Environmental Biology and Chemistry, University of Bari, Bari, Italy \\ ${ }^{\mathrm{c}}$ Human Physiology Unit, Department of Physiology, Faculty of Medicine, University of Pavia, Pavia, Italy
}

\section{A R T I C L E I N F O}

\section{Article history:}

Received 25 September 2009

Received in revised form 19 January 2010

Accepted 19 January 2010

\section{Keywords:}

Muscle atrophy

Muscle plasticity

Oxidative stress

Muscle excitability

Sarcolemma chloride conductance

Antioxidant

\begin{abstract}
A B S T R A C T
Oxidative stress was proposed as a trigger of muscle impairment in various muscle diseases. The hindlimb-unloaded (HU) rodent is a model of disuse inducing atrophy and slow-to-fast transition of postural muscles. Here, mice unloaded for 14 days were chronically treated with the selective antioxidant trolox. After HU, atrophy was more pronounced in the slow-twitch soleus muscle (Sol) than in the fast-twitch gastrocnemius and tibialis anterior muscles, and was absent in extensor digitorum longus muscle. In accord with the phenotype transition, HU Sol showed a reduced expression of myosin heavy chain type 2A (MHC-2A) and increase in MHC-2X and MHC-2B isoforms. In parallel, HU Sol displayed an increased sarcolemma chloride conductance related to an increased expression of $\mathrm{ClC}-1$ channels, changes in excitability parameters, a positive shift of the mechanical threshold, and a decrease of the resting cytosolic calcium concentration. Moreover, the level of lipoperoxidation increased proportionally to the degree of atrophy of each muscle type. As expected, trolox treatment fully prevented oxidative stress in HU mice. Atrophy was not prevented but the drug significantly attenuated Sol phenotypic transition and excitability changes. Trolox treatment had no effect on control mice. These results suggest possible benefits of antioxidants in protecting muscle against disuse.
\end{abstract}

(c) 2010 Elsevier Ltd. All rights reserved.

\section{Introduction}

Adult skeletal muscles contain slow- and fast-twitch fibers according to their speed of contraction. Slow-twitch myofibers express the slow type- 1 isoform of myosin heavy chain (MHC1 ), whereas fast-twitch myofibers may express three types of fast MHC (MHC-2A, 2B or 2X) [1]. Differential expression of MHC isoforms affects specific force and is a major determinant of the maximal velocity of shortening of individual muscle fiber. Noteworthy, adult muscle fibers can adapt their phenotype to modified functional requests by expressing different levels or forms of proteins involved in the control of muscle excitability,

Abbreviations: HU, hindlimb unloading; Sol, soleus muscle; Gas, gastrocnemius muscle; EDL, extensor digitorum longus muscle; TA, tibialis anterior muscle; MHC, myosin heavy chain; MDA, malondialdehyde; $\mathrm{gCl}$, chloride conductance of sarcolemma at rest; gK, potassium conductance of sarcolemma at rest; restCa, resting cytosolic calcium concentration; MT, mechanical threshold; CSA, cross-sectional area.

* Corresponding author. Tel.: +39080 54428 01; fax: +39 0805442801.

E-mail address: conte@farmbiol.uniba.it (D.C. Camerino). excitation-contraction coupling, energy metabolism, and contractile function. For instance a slow-to-fast phenotype transition is a hallmark of muscle adaptation to reduced neuromuscular activity [1]. A slow-to-fast transition was also observed in hindlimb muscles of rats treated with a $\beta 2$-adrenoceptor agonist, and was associated with muscle function impairment [2]. Thus such phenotypic functional changes likely contribute to disuse-induced muscle impairment, and consequently represent a target for prevention. Importantly, the promotion of slow, oxidative fibers has been shown to mitigate the progression of muscular dystrophy and proposed as a possible countermeasure to overcome muscle dysfunction associated with insulin-resistant states [3].

In the hindlimb-unloaded (HU) rat, a model for muscle disuse that mimics the effects of microgravity, a partial slow-to-fast fiber type transition occurs in the postural, slow-twitch soleus (Sol) muscle. After 14 days of HU, the proportion of fast MHCpositive fibers may reach $\sim 40 \%$ in the Sol muscle compared to $<15 \%$ in control rats [4]. These effects are slowly reversed after several weeks of reloading [5]. Little is known about the triggers and molecular mechanisms responsible for disuse-induced fiber type transition. We previously described the effects of HU on ion channels that are critical for phenotype-specific sarcolemma excitability 
and contractile function, and may contribute to modulation of gene transcription [4,6-9]. For example, the macroscopic chloride conductance $(\mathrm{gCl})$, which is by far the largest component conductance of adult muscle at resting potential and consequently a key determinant of muscle excitability, is higher in fast-twitch compared to slow-twitch muscle fibers. In accord with fiber type transition, the $\mathrm{gCl}$ increases in $\mathrm{HU}$ Sol muscle toward a value more similar to that measured in fast-twitch muscles [4]. Such an effect alters sarcolemma excitability in Sol muscle fibers and may contribute to impair resistance to fatigue and to trigger fiber type transition [8].

Besides phenotype transition, HU Sol muscles undergo a significant atrophy due mainly to an increased protein degradation. Muscle atrophy is a severe clinical symptom encountered in various diseases, which has thus deserved much more attention than fiber type transition. Oxidative stress has been observed in many of these diseases and has been recently pinpointed as a possible causative factor for muscle atrophy $[10,11]$. Whether oxidative stress may influence fiber type transition is however completely unknown.

In the present study, we studied the effects of 14 days $\mathrm{HU}$ on hindlimb muscles of 6-month-old mice as well as the effects of concomitant treatment with the potent and selective antioxidant trolox. Muscle atrophy, phenotype, and function were assessed in Sol and gastrocnemius (Gas) muscles using multidisciplinary approaches. Some parameters were also measured in the extensor digitorum longus (EDL) and tibialis anterior (TA) muscles. By measuring lipoperoxidation, we also verified whether oxidative stress occurs in HU mouse muscles and whether it is prevented by trolox treatment. Indeed administration of trolox was previously shown to attenuate diaphragm muscle atrophy induced by mechanical ventilation in rats [12].

The main results showed that the degree of atrophy in each muscle was parallel to the level of oxidative stress, but no beneficial effect of trolox treatment was found on muscle atrophy. Nevertheless trolox, which neutralized oxidative stress in all muscles, balanced the phenotype transition. These results suggest the possibility to use antioxidants to ameliorate phenotype-dependent, disuse-induced muscle functional impairment.

\section{Material and methods}

\subsection{Animal care and hindlimb unloading}

Experiments were approved by the Italian Health Department and complied to the Italian guidelines for the use of laboratory animals, which conform with the European Community Directive published in 1986 (86/609/ECC). Six-month-old male C57BL mice weighting 27-32 g (Charles River Laboratories, Calco, Italy) were randomly assigned to CTRL (control mice), HU (hindlimb-unloaded for 14 days), TRO (treated with trolox for 3 weeks) and HUTRO (treated with trolox for 3 weeks and hindlimb unloaded for the last two 14 days) groups. The CTRL mice were maintained free in single cages for 14 days. To induce muscle unloading, the animals of HU group were suspended individually in special cages for 2 weeks using a method similar to that used previously for $\mathrm{HU}$ rats [8]. A thin string was linked at one extremity to the tail by sticking plaster and at the other extremity to the top of the cage. The length of the string was adjusted to allow the animals moving freely on the forelimbs, while the body was inclined at $30-40^{\circ}$ from the horizontal plane. All mice had water ad libitum and received $8 \mathrm{~g}$ a day of standard rodent chow. The food remaining on the day after was weighted to calculate daily food consumption. The TRO and HUTRO mice received daily, 6 days a week, an intraperitoneal injection of $0.25 \mathrm{ml}$ of a $1 \mathrm{M} \mathrm{NaHCO}_{3}$ solution containing $5 \mathrm{~g} / \mathrm{L}$ trolox, corresponding to $\sim 45 \mathrm{mg} / \mathrm{kg} /$ day dose (The dose was double on the sixth day to maintain drug level along the seventh day). Trolox [( \pm )-6-hydroxy-2,5,7,8-tetramethylchromane-2-carboxylic acid, Sigma, Milan, Italy] is a water-soluble vitamin E analogue that freely crosses cell membranes [13]. The drug displays a very high antioxidant capacity and may lack antioxidant-unrelated side effects, so that it is widely used as a standard compound in antioxidant capacity assays. Accordingly, such a treatment was able to prevent lipid perodixation (see results). At the end of suspension, the mice were unfastened from the string and deeply anesthetized by intraperitoneal injection of urethane $(1.2 \mathrm{~g} / \mathrm{kg}$ body weight) to allow removing of soleus (Sol), gastrocnemius (Gas), tibialis anterior (TA), and extensor digitorum longus (EDL) muscles. Muscles were used immediately for the electrophysiological experiments or frozen in liquid nitrogen and stored at $-80^{\circ} \mathrm{C}$ for other studies. After surgery, animals were euthanized by an overdose of urethane. For measuring the markers of oxidative stress, the mice were sacrificed by cervical dislocation and the muscles removed and frozen promptly.

\subsection{Ex vivo electrophysiological studies}

Soleus, EDL, or Gas muscles were fixed by tendons to a glass rod immersed in normal (NP) or chloride-free physiological solution maintained at $30^{\circ} \mathrm{C}$ and perfused with $95 \% \mathrm{O}_{2} / 5 \% \mathrm{CO}_{2}$ [4]. The NP solution contained (in $\mathrm{mM}$ ): $\mathrm{NaCl} 148, \mathrm{KCl} 4.5, \mathrm{CaCl}_{2} 2.0$, $\mathrm{MgCl}_{2}$ 1.0, $\mathrm{NaHCO}_{3}$ 12.0, $\mathrm{NaH}_{2} \mathrm{PO}_{4}$ 0.44, glucose 5.5, and pH 7.2. The chloride-free solution was prepared by equimolar substitution of methylsulfate salts for $\mathrm{NaCl}$ and $\mathrm{KCl}$ and nitrate salts for $\mathrm{CaCl}_{2}$ and $\mathrm{MgCl}_{2}$. The cable parameters of myofiber sarcolemma were determined from the electrotonic potentials elicited by squarewave hyperpolarizing current pulse of $100-$ ms duration, using two intracellular microelectrodes in current-clamp mode, as previously described [14]. The membrane conductance is calculated from the values of input resistance, space constants and time constant and assuming a myoplasmic resistivity of $125 \Omega \mathrm{cm}$. The mean chloride conductance $(\mathrm{gCl})$ is calculated as the mean total membrane conductance ( $\mathrm{gm}$ ) measured in NP solution minus the mean potassium conductance gK measured in chloride-free solution.

Sarcolemma excitability parameters were determined by applying $100 \mathrm{~ms}$-long depolarizing current pulses of increasing amplitude to elicit first a single action potential (AP) then a train with the maximal number of APs. The membrane potential was held at $-80 \mathrm{mV}$ between test pulses. The excitability parameters, determined off-line on digital AP recordings, were the current threshold to elicit the first AP (or Rheobase current, $I_{\mathrm{th}}$ ), the latency of the AP (Lat), the AP amplitude (APA), the maximal number of elicitable $\mathrm{AP}$ ( $\mathrm{N}$ spikes), and the ratio between $I_{\text {th }}$ and the current threshold needed to elicit more than one $\mathrm{AP}\left(I_{\mathrm{th}} / I_{2}\right)$. The Lat is inversely correlated to the $\mathrm{gCl}$, whereas the other parameters depend on various ion channels/carriers.

The mechanical threshold (MT) for contraction was determined using a two-microelectrode point voltage clamp method in the presence of $3 \mu \mathrm{M}$ tetrodotoxin, as described previously [4]. The holding potential was set to $-90 \mathrm{mV}$. Depolarizing current pulses of various durations $(5-500 \mathrm{~ms})$ were applied at a frequency of $0.3 \mathrm{~Hz}$, while the impaled fiber was continuously inspected with a stereomicroscope. The command voltage was increased until contraction was visible and the threshold membrane voltage was read at this time from a digital sample-and-hold voltmeter. The mean threshold voltage $V(\mathrm{mV}) \pm$ S.E.M. ( $n$ fibers) was plotted as a function of the pulse duration $t(\mathrm{~ms})$, and the relationship was fit using a nonlinear least squares algorithm with the equation,

$V(t)=\frac{\left[-90-R \cdot \exp \left(-t / t_{R}\right)\right]}{\left[1-\exp \left(-t / t_{R}\right)\right]}$

where $R(\mathrm{mV})$ is the Rheobase voltage and $t_{R}(\mathrm{~ms})$ the time constant to reach $R$. The MT values were expressed as the calculated $R$ value along with the standard error of the fit. 


\subsection{Quantitative real time $P C R$}

For each Sol and EDL muscle sample, the total RNA was isolated by using RNeasy Fibrous Tissue Mini Kit (QIAGEN). RNA was quantified by using a spectrophotometer (ND-1000 NanoDrop, Thermo Scientific), and $400 \mathrm{ng}$ were used for reverse transcription. Synthesis of cDNA was performed by using random hexamers (annealed $10 \mathrm{~min}, 25^{\circ} \mathrm{C}$ ) and Superscript II reverse transcriptase (Invitrogen, Life Technologies) incubated at $42^{\circ} \mathrm{C}$ for $50 \mathrm{~min}$. We identified the available mouse sequence for ClC-1 (NM_013491) and $\beta$-actin (NM_007393). Fluorescently labelled TaqMan MGB probes (Applied Biosystems) for $\mathrm{ClC}-1$ and $\beta$-actin were designed by using Primer Express (Applied Biosystems) to amplify respectively 59- and 71-bp products encompassing each probe annealing site. To achieve a high level of specificity and to avoid detection of genomic DNA, the probe was designed to span exon-exon junctions for each gene. Specific primer and probe sequences for each gene are available on request. The $\beta$-actin gene was tested as internal control, together with the commercial housekeeping genes hypoxanthine guanine phosphoribosyl transferase 1 (HPRT1) and $\beta 2$-microglobulin (Applied Biosystem). Triplicate reactions were carried out in parallel for each individual muscle sample, and the results were compared with a gene-specific standard curve. The HPRT1 gene was individuated as the more suitable housekeeping gene by using GeNorm 3.4 software, and was used for normalization of $\mathrm{ClC}-1$ signal. Qualitatively similar results were obtained using $\beta$-actin (not shown).

\subsection{Determination of the cytosolic calcium concentration at rest (restCa)}

The resting cytosolic $\mathrm{Ca}^{2+}$ concentration (restCa) was determined in freshly, mechanically dissected muscle fibers using a QuantiCell 900 fluorescence imaging system (Visitech International, Sunderland, UK), as previously described [7]. Briefly, small bundles of 5-10 fibers were dissected tendon to tendon from Sol, Gas, and EDL muscles. The bundles were incubated for $60-90 \mathrm{~min}$ at $30^{\circ} \mathrm{C}$ in NP solution (in $\mathrm{mM}, 148 \mathrm{NaCl}, 4.5 \mathrm{KCl}, 2.5 \mathrm{CaCl}_{2}, 1 \mathrm{MgCl}_{2}$, $12 \mathrm{NaHCO}_{3}, 0.44 \mathrm{NaH}_{2} \mathrm{PO}_{4}, 5.5$ glucose, and pH 7.3), supplemented with $5 \mu \mathrm{M}$ of acetoxymethyl ester of Fura- 2 mixed to $10 \%(\mathrm{v} / \mathrm{v})$ pluronic F-127 (Molecular Probes, Leiden, The Netherlands). After incubation, the cells were washed with NP solution and mounted in a modified RC-27NE recording chamber (Warner Instruments, Hamden, CT). Tendons of the bundles were attached by hair loops, one extremity to a fixed tube, the other to a mobile one. The chamber was accommodated on the stage of an inverted Eclipse TE300 microscope equipped with a $\times 40$ pan-fluor objective (Nikon, Tokyo, Japan). The recording chamber was continuously perfused with NP solution at a constant rate of $\sim 4 \mathrm{ml} / \mathrm{min}$.

Fiber integrity was verified by assessing contractile activity under $\times 400$ magnification in response to a depolarizing solution containing $100 \mathrm{mM} \mathrm{K}^{+}$. Then the mean sarcomere length was adjusted to $\sim 2.5 \mu \mathrm{m}$. The mean resting background-corrected ratio $(340 \mathrm{~nm} / 380 \mathrm{~nm})$ values were determined for each fiber of the bundle by manually demarcating fiber boundaries using QC2000 software. This fluorescence ratio was converted off-line to restCa by using the calibration parameters determined in each muscle, using the equation restCa $=\left(R-R_{\min }\right) /\left(R_{\max }-R\right) \cdot K_{\mathrm{D}} \cdot \beta$, where $R$ is the 340 to $380 \mathrm{~nm}$ fluorescence ratio; $K_{\mathrm{D}}$ is the affinity constant of Fura-2 for $\mathrm{Ca}^{2+}$ given by the manufacturer, i.e. $145 \mathrm{nM}$ (Molecular Probes), and $\beta, R_{\min }$, and $R_{\max }$ are calibration parameters determined in ionomycin-permeabilized fibers bathed in NP solution for the calculation of $R_{\max }$ or in $\mathrm{Ca}^{2+}$-free solution for the calculation of $R_{\min }$ [15]. The $\beta$ value was calculated as the ratio of fluorescence

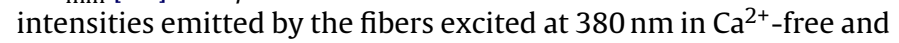
NP solutions. The calibration parameters were measured for every muscle types in each experimental condition, because they may be different between muscle types and may change critically as a function of muscle state [7,16].

\subsection{Gas muscle CSA analysis}

The cross-sectional area (CSA) of individual muscle fibers was determined on serial transverse sections of Gas muscle samples collected from 3 mice in each experimental condition. The muscles were included in OCT embedding medium and frozen in liquid nitrogen. Serial transverse sections $(10 \mu \mathrm{m}$ thick) were cut using a Leica CM 1850 cryostat, and stained for haematoxylin-eosin. Images of the stained sections were captured from a light microscope (Leica DMLS) and transferred to a personal computer using a video camera (Leica DFC 280). Cross-sectional areas of fibers was measured with Scion Image Analysis Software (NIH, Bethesda, MD, USA) and expressed in $\mu \mathrm{m}^{2}$.

\subsection{Analysis of MHC isoform content}

The MHC isoform composition of each muscle sample was determined on $8 \%$ polyacrylamide slab gels after denaturation in SDS (SDS-PAGE) with a procedure derived from that described by Talmadge and Roy [17]. Muscle samples were dissolved in lysis buffer (Tris $62.5 \mathrm{mM}$, pH 6.8, SDS 2.3\%, glycerol 10\%, 2-mercaptoethanol $5 \%$ ) [18]; and the protein concentration was determined with a protein assay kit (RC DC Protein Assay, Biorad) based on the Lowry protocol [19]. About $6 \mu \mathrm{g}$ of each muscle sample were loaded onto the gels. Electrophoresis was run for $2 \mathrm{~h}$ at $200 \mathrm{~V}$ and then for $24 \mathrm{~h}$ at 250 V. Gels were stained with Coomassie blue for identification of protein bands. Four bands were separated in the region of $200 \mathrm{kDa}$, corresponding, in order of migration from the fastest to the slowest, to MHC-1 or slow, MHC-2B, MHC-2X and MHC-2A. The MHC isoforms bands have been previously identified on the base of Western blot analysis using anti-MHC monoclonal antibodies [20]. The relative proportion of the $\mathrm{MHC}$ isoforms was determined by densitometric analysis of the bands. An example of SDS-PAGE separation of MHC isoforms is shown in Fig. 3.

To assess the percent of hybrid fibers, which is believed to increase following disuse, individual muscle fibers were dissected from soleus muscles and characterized on the basis of their MHC isoform content using the same SDS-PAGE protocol employed to study MHC isoforms distribution in whole muscle samples. One hundred muscle fibers were studied from both CTRL and HU Sol muscles. No such analysis was performed in EDL, TA and Gas as they almost solely express MHC-B.

\subsection{Measurement of malondialdehyde (MDA) levels}

We determined the levels of malondialdehyde (MDA), as an indicator of oxidative stress, in Sol, Gas, TA, and EDL muscles of CTRL, HU, and HUTRO mice. The mice were killed by cervical dislocation. Very quickly, the muscles were dissected, weighted, and frozen in liquid nitrogen. The whole muscles were homogenized in $3 \mathrm{ml}$ (Gas muscle), $1 \mathrm{ml}$ (TA), or $0.8 \mathrm{ml}$ (Sol and EDL) ice-cold Tris- $\mathrm{HCl}$ $(20 \mathrm{mM})$ solution. The homogenate was centrifuged at $4000 \mathrm{rpm}$ at $4{ }^{\circ} \mathrm{C}$ for $10 \mathrm{~min}$, and the supernatant was collected and frozen at $-20^{\circ} \mathrm{C}$ until the day of assays. The MDA level, which reflects the level of lipid peroxidation, was measured by fluorescence assay of thiobarbituric acid reactive substance (TBARS) formation following the indications of a commercial kit (OXItek, ZeptoMetrix Corp., Buffalo, NY). Fluorescence measurements were performed in triplicate using a 96 -wells plate reader (Victor ${ }^{3} \mathrm{~V}$ multilabel counter, PerkinElmer). The measured MDA levels were normalized with respect to homogenization volume and muscle weight. 

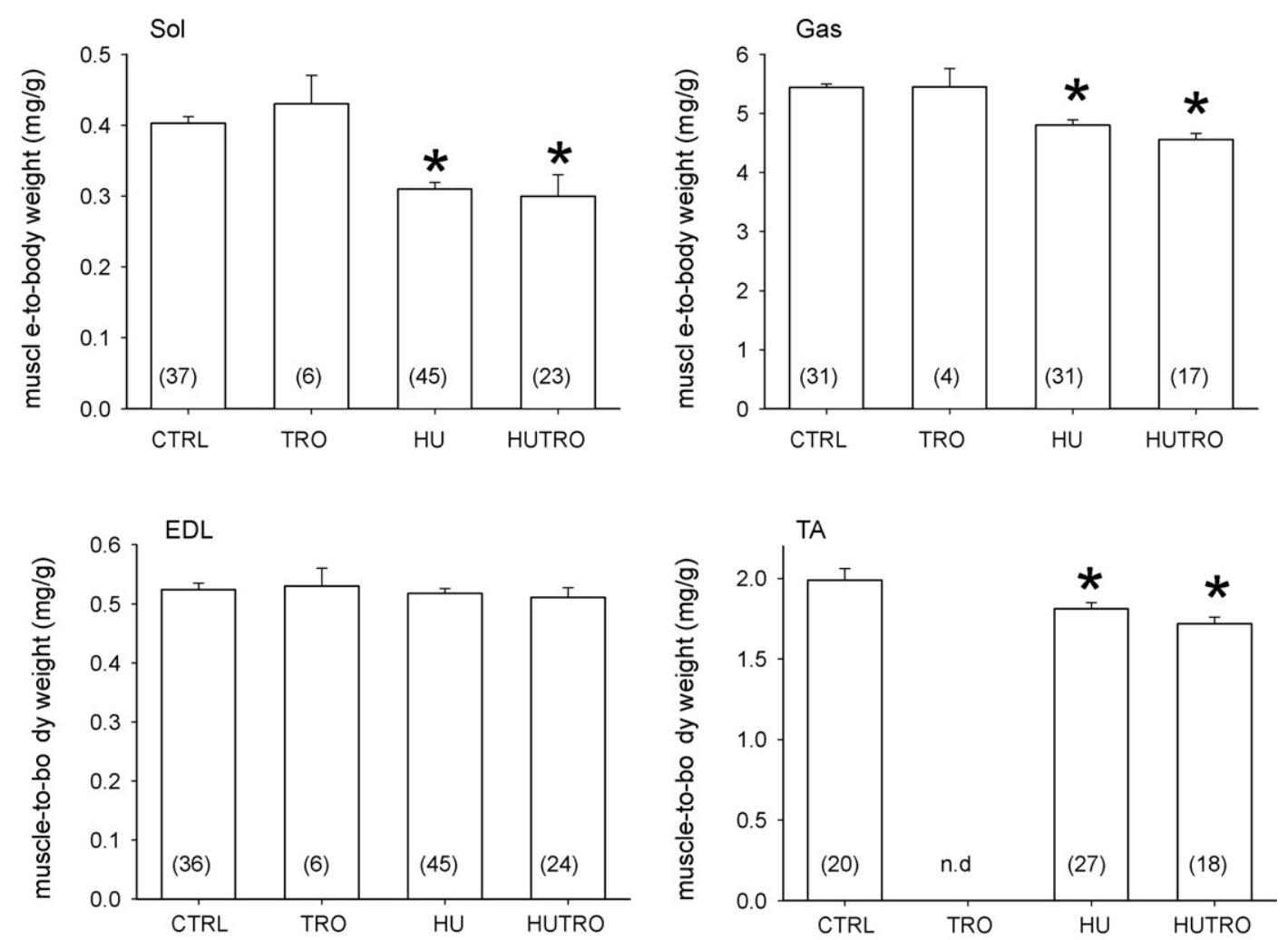

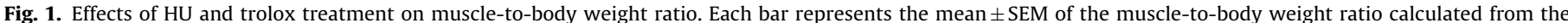

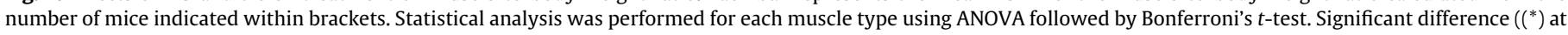
least $P<0.02$ ) was found versus CTRL and TRO.

\subsection{Statistical analysis}

Data were expressed as mean \pm S.E.M. The number of mice in each group can vary substantially (e.g. Fig. 1), because not all the parameters were determined in each group, muscle type, or mouse. For instance, the number of TRO mice was lower than the number of mice used for other conditions because trolox treatment was used only to verify whether the drug may affect parameters that were also targets of $\mathrm{HU}$, such as muscle weight, MHC expression, and $\mathrm{gCl}$, especially in Sol and Gas muscles. Statistical significance of the differences between means of two groups was assessed by Student's $t$-test. For comparison between more than two groups, ANOVA followed by Bonferroni's $t$-test was used. A probability of less than $5 \%$ was considered significant $(P<0.05)$.

\section{Results}

\subsection{Effects of HU and trolox on mouse health and behavior}

Our 2-years experience with HU mice, which were used for the present study and other unpublished studies, indicates that HU produced significant stress in 4 out of 64 mice (6.3\%), as evidenced by mouse refusal to eat during the first 2 days of suspension. The four stressed animals were unfastened on the second day and discarded from the study. The remaining HU mice were examined daily over the entire HU period for behavior, cleanliness, aspect of hairs and eyes, and food and water consumption. No sign of stress was evidenced. Daily food consumption over 14 days did not differ significantly between 15 CTRL and $15 \mathrm{HU}$ mice $(4.47 \pm 0.14 \mathrm{~g} /$ day versus $4.68 \pm 0.15 \mathrm{~g} /$ day, respectively). The control 6-month-old mice showed no variation in body weight over 14 days $(-0.30 \pm 0.23 \mathrm{~g}, n=31)$, whereas age-matched mice showed a little but significant weight loss during 14 days of $\mathrm{HU}$
$(-1.07 \pm 0.23 \mathrm{~g}, n=54, P<0.03$ versus CTRL with unpaired Student's $t$-test). The ground-based (TRO) or hindlimb-unloaded (HUTRO) mice receiving trolox injection daily showed no difference with CTRL and HU non-treated animals, respectively. Two out of 28 HUTRO mice were discarded from the study because they refused to eat during suspension. Daily food consumption over 14 days was $4.54 \pm 0.24 \mathrm{~g} /$ day $(n=6)$ for TRO mice and $4.70 \pm 0.15 \mathrm{~g} /$ day $(n=26)$ for HUTRO mice. Body weight variation over 14 days was $-0.49 \pm 0.47 \mathrm{~g}(n=7)$ for TRO mice and $-0.95 \pm 0.30 \mathrm{~g}(n=26)$ for HUTRO mice.

\subsection{Effects of HU and trolox on mouse hindlimb muscle weight and phenotype}

We collected muscle weight data from a large number of mice, as an index of muscle atrophy [21]. The Sol, Gas, and TA muscles showed significant atrophy after 14 days of HU, as evidenced by the reduction of both the muscle weight (not shown) and the muscleto-body weight ratio (Fig. 1). Atrophy was more pronounced in Sol (-24.4\%) compared to Gas (-11.9\%) and to TA (-9.5\%). In contrast, no significant change in the EDL muscle weight and EDL muscleto-body weight ratio was observed, confirming lack of atrophy in this muscle, as in the rat [4]. Compared to non-treated mice, trolox treatment had no effect on muscle-to-body weight ratio either in CTRL or HU mice (Fig. 1). Analysis of cross-sectional area of individual muscle fibers of Gas muscles collected from CTRL, HU, and HUTRO mice confirms HU-induced myofiber atrophy and the lack of trolox treatment benefit in contrasting HU-induced atrophy (Fig. 2).

It is widely recognized that Sol muscles of $\mathrm{HU}$ rats also undergo a partial slow-to-fast shift of MHC expression [4,5]. Thus we determined the MHC isoform content in the Sol muscle of CTRL and HU mice by electrophoretic SDS-PAGE separation of MHC isoforms and subsequent densitometric analysis of myosin heavy chain bands 
CTRL

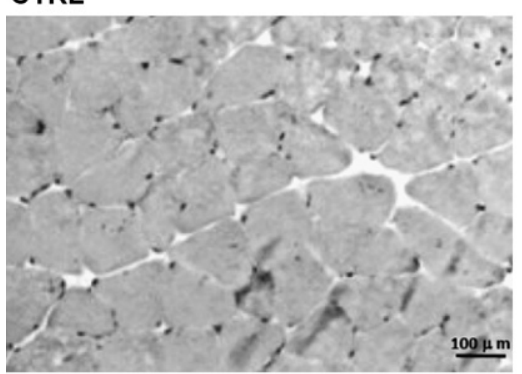

HUTRO

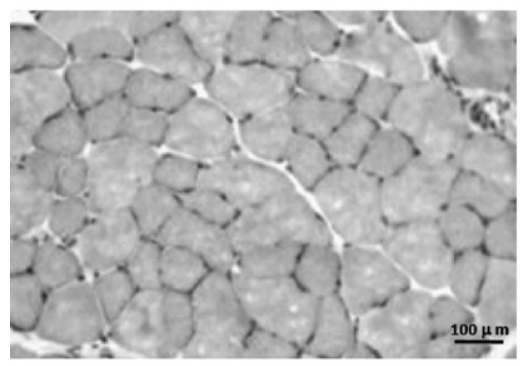

HU
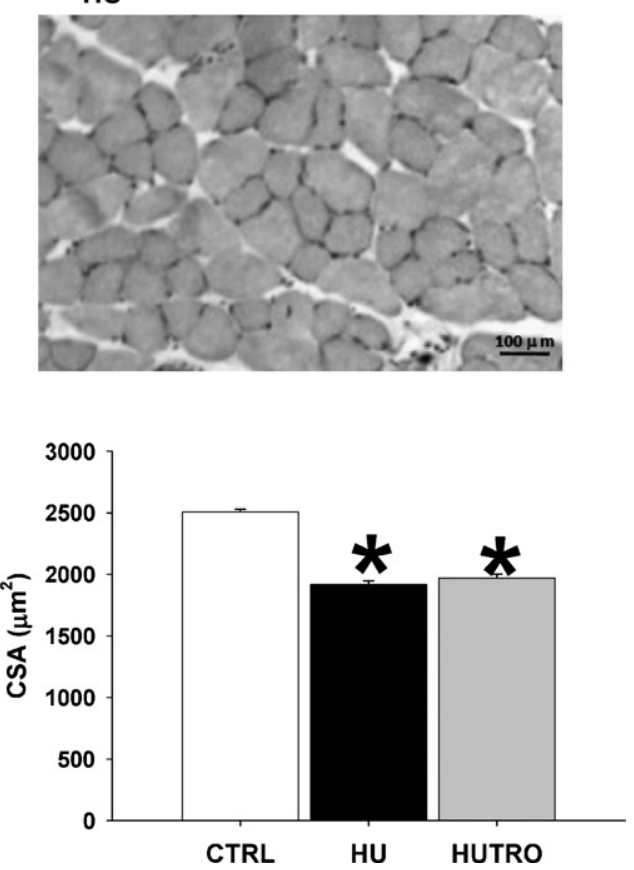

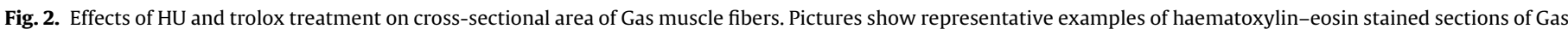

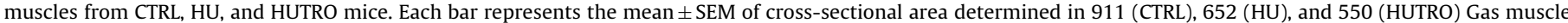

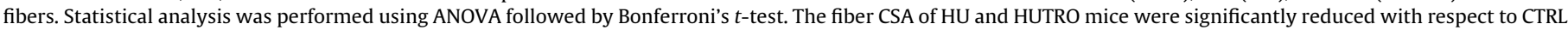
mice $(P<0.001)$.

(Fig. 3). Compared to CTRL rats that express $\sim 90 \%$ of the slow MHC1 isoform and only $10-15 \%$ of the fast MHC-2A isoform [4], the Sol muscle of mice appears as a mixed muscle with only $~ 39 \%$ of the slow MHC- 1 isoform, $~ 50 \%$ of the fast MHC-2A isoform, and $\sim 11 \%$ of the fast MHC-2X isoform (Fig. 3). After 14 days of HU, there was no change in MHC-1 content, whereas the proportion of MHC-2A decreased to $32 \%$ of the total MHC content, the MHC-2X content increased to $25 \%$, and a little proportion of MHC-2B could be measured. Thus, on the basis of MHC isoform content, the Sol muscle of mice became faster after $\mathrm{HU}$. In contrast, little or no effect of HU was found on MHC content in the fast-twitch TA, EDL, and Gas muscles. Since it has been shown that the percent of hybrid fibers increases in disused rat soleus muscle [5], such parameter was determined in HU Sol muscles of mice by studying the MHC isoform content of individual myofibers by SDS-PAGE. The percentage of hybrid fibers was found to be higher in $\mathrm{HU}$ ( $50.6 \%$ pure fibers, $49.4 \%$ hybrid fibers, $n=100$ fibers) than CTRL mice $(80.7 \%$ pure fibers, $19.3 \%$ hybrid fibers, $n=100$ fibers). No such analysis was performed on Gas, TA and EDL muscles due to their almost homogeneous MHC-2B content. Chronic treatment of control mice with trolox had no effect on MHC expression in either Sol or Gas muscles (Fig. 3). In contrast, the re-distribution of MHC isoforms was significantly less pronounced in Sol muscles of HUTRO mice compared to HU mice. This result suggests that trolox was able to partially prevent the shift of Sol muscle phenotype induced by HU.

\subsection{Effects of $H U$ and trolox on the resting conductances, chloride channel ClC-1 mRNA expression, and excitability of sarcolemma}

We have previously observed, using rats, that the resting chloride conductance $(\mathrm{gCl})$ is about 1.8 times higher in the fast-twitch EDL muscle with respect to the slow-twitch Sol muscle [4]. After 14 days of $\mathrm{HU}$, the $\mathrm{gCl}$ increased by $30-40 \%$ in Sol muscle fibers of rats in accordance with the partial slow-to-fast transition of muscle phenotype $[5,8]$. In contrast, no effect of HU was observed on the resting potassium conductance $(\mathrm{gK})$ in rat muscles.
In CTRL mice, the $\mathrm{gCl}$ was $1344 \pm 44 \mu \mathrm{S} / \mathrm{cm}^{2}$ in Sol muscle fibers $\left(N\right.$ mice $/ n$ fibers $=8 / 80$ ) and $2423 \pm 96 \mu S / \mathrm{cm}^{2}$ in EDL muscle fibers (4/27). In the Gas muscle fibers of CTRL mice, the $\mathrm{gCl}$ was $2477 \pm 65 \mu \mathrm{S} / \mathrm{cm}^{2}(3 / 28)$. Chronic treatment of control mice with trolox had no effect on the $\mathrm{gCl}$ of Sol and Gas muscle fibers. After 14 days of $\mathrm{HU}$, the $\mathrm{gCl}$ increased significantly by $36 \pm 5 \%$ in Sol muscle fibers and by $12 \pm 3 \%$ in Gas muscle fibers (Fig. 4). No significant change was found in the EDL muscle fibers after HU. Trolox treatment of control mice had no effect on the $\mathrm{gCl}$ of Sol muscle fibers. Remarkably, HUTRO mice did not show any change of the $\mathrm{gCl}$ in both Sol and Gas muscle fibers with respect to CTRL mice, indicating that trolox treatment was able to fully prevent the disuse-associated change in $\mathrm{gCl}$.

In CTRL mice, the sarcolemma potassium conductance at rest was significantly (at least $P<0.05$ with ANOVA followed by Bonferroni's $t$-test) higher in the Sol muscle $\left(485 \pm 29 \mu \mathrm{S} / \mathrm{cm}^{2}\right.$, $N=6 / n=44)$ compared to $\operatorname{EDL}\left(378 \pm 27 \mu \mathrm{S} / \mathrm{cm}^{2}, N=3 / n=21\right)$ and Gas muscles $\left(328 \pm 23 \mu \mathrm{S} / \mathrm{cm}^{2}, N=3 / n=21\right)$. Neither HU nor trolox treatment showed significant effect on the gK in Sol, EDL, and Gas muscle fibers (not shown).

Using Northern blot analysis, we previously showed that the increased $\mathrm{gCl}$ in rat Sol muscle fibers after 7 or 21 days of $\mathrm{HU}$ is related to an increased expression of the CLCN1 gene encoding the voltage-gated chloride channel $\mathrm{ClC}-1$ [4]. In the present study, we determined $\mathrm{ClC}-1$ channel mRNA levels in Sol and EDL muscles of CTRL, HU, and HUTRO mice using quantitative real time PCR. Similar to other studies that used Northern blot analysis [22], the Sol muscle express about one-third of $\mathrm{ClC}-1$ mRNA compared to EDL muscle in CTRL mice (Fig. 4b). After 14 days HU, ClC-1 mRNA expression significantly increased by $37.5 \%$ in Sol muscle in accord with the increased $\mathrm{gCl}$. The treatment with trolox fully prevented the increase of $\mathrm{ClC}-1$ expression in Sol muscle in HUTRO mice. No significant effect of HU and trolox was found in EDL muscle.

We also tested the acute effects of ex vivo application of trolox $(300 \mu \mathrm{M})$ to Sol muscle fibers of HU mice. In these conditions, the total sarcolemma conductance (gm) was $2406 \pm 170 \mu \mathrm{S} / \mathrm{cm}^{2}$ 

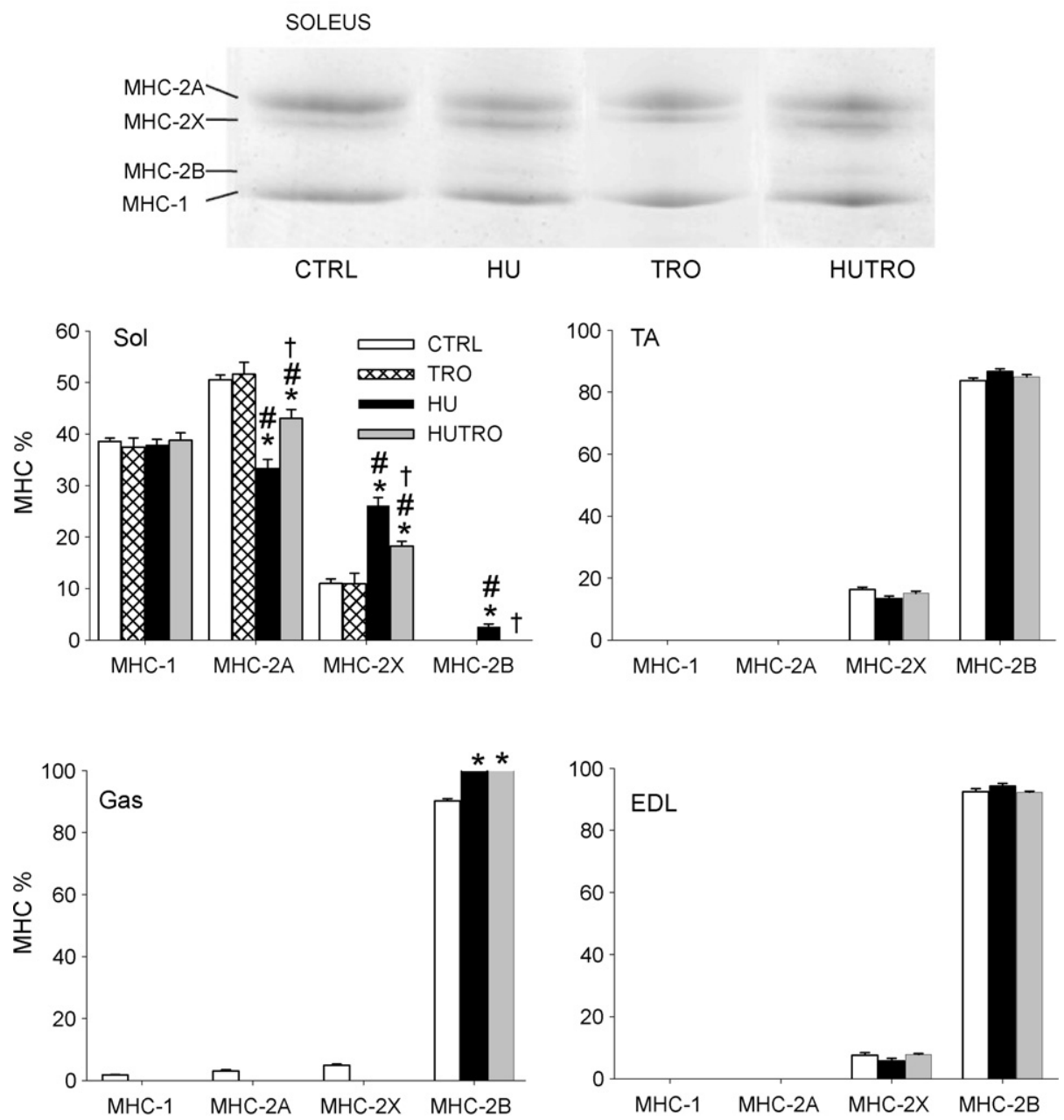

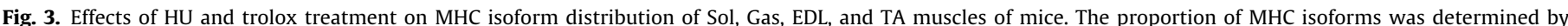

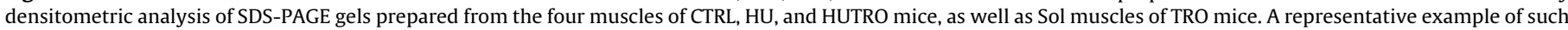

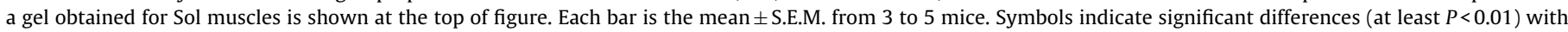
respect to CTRL $\left({ }^{*}\right)$, TRO (\#), and HU $(\dagger)$, determined by ANOVA followed by Bonferroni's $t$-test.

( $n=11)$ and $2259 \pm 177 \mu \mathrm{S} / \mathrm{cm}^{2}(n=14)$ before and $20-60$ min after trolox application, respectively. Thus trolox showed no significant acute effect on the gCl. Nevertheless, we cannot definitely exclude that trolox may exert indirectly an acute action on the $\mathrm{gCl}$ in vivo, which could not be reproduced ex vivo.

The $\mathrm{gCl}$ is a critical determinant of sarcolemma excitability [8]. In control mice, phenotypic differences between Sol and EDL muscles include a smaller rheobase current $\left(I_{\mathrm{th}}\right)$, a longer latency, which is notably inversely proportioned to the $\mathrm{gCl}$, and lower AP amplitude in the slow-twitch muscle fibers (Table 1 ). Thus many of the phenotypic differences observed in the rat are also present in the mouse, although less accentuated as expected from the mixed nature of the mouse Sol muscle. In the mouse, no difference was found between Sol and EDL muscles in the maximum number of elicitable action potentials ( $N$ spikes) or in the $I_{\text {th }} / I_{2}$ ratio. A smaller rheobase current in the Sol muscle may allow this muscle to be tonically active to bear normal gravity loading.

After 14 days of $\mathrm{HU}$, the Sol muscle fibers showed a shorter latency, as expected from the greater $\mathrm{gCl}$, an increased APA, a reduced $I_{\text {th }} / I_{2}$ ratio, which is suggestive of a reduced excitability of HU Sol muscle with respect to control Sol. Importantly, the treatment with trolox prevented the change in latency, in accord with the prevention of $\mathrm{gCl}$ change, as well as in APA and $I_{\mathrm{th}} / I_{2}$ ratio in HU Sol muscle.

\subsection{Effects of HU and trolox on the mechanical threshold}

The MT, that is the smallest voltage able to elicit a contraction, is an integrative measure of the excitation-contraction coupling mechanism (Fig. 5). The MT was determined at different current pulse durations in order to obtain a threshold-duration relation-

Table 1

Effects of disuse on excitability parameters in EDL and Sol muscles of control mice and Sol muscles of HU and HUTRO mice.

\begin{tabular}{llllll}
\hline Muscle & $I_{\text {th }}(\mathrm{nA})$ & Lat $(\mathrm{ms})$ & APA $(\mathrm{mV})$ & $I_{\text {th }} / I_{2}$ & N spikes \\
\hline EDL & $102 \pm 11$ & $5.3 \pm 0.5$ & $92.8 \pm 3.6$ & $0.76 \pm 0.07$ & $4.1 \pm 0.4$ \\
$(N=2)$ & $(n=12)$ & $(n=12)$ & $(n=12)$ & $(n=6)$ & $(n=9)$ \\
Sol & $63.3 \pm 6.2^{\mathrm{a}}$ & $7.0 \pm 0.6^{\mathrm{a}}$ & $72.9 \pm 3.3^{\mathrm{a}}$ & $0.75 \pm 0.03$ & $3.8 \pm 1.0$ \\
$(N=2)$ & $(n=10)$ & $(n=10)$ & $(n=10)$ & $(n=8)$ & $(n=10)$ \\
HU & $58.4 \pm 5.5^{\mathrm{a}}$ & $5.9 \pm 0.3^{\mathrm{b}}$ & $88.0 \pm 2.8^{\mathrm{b}}$ & $0.54 \pm 0.04^{\mathrm{a}}, \mathrm{b}$ & $3.3 \pm 0.4$ \\
$(N=4)$ & $(n=25)$ & $(n=25)$ & $(n=25)$ & $(n=20)$ & $(n=22)$ \\
HUTRO & $59.7 \pm 5.0^{\mathrm{a}}$ & $6.7 \pm 0.6^{\mathrm{a}}$ & $78.4 \pm 3.4^{\mathrm{a}}$ & $0.66 \pm 0.08$ & $3.4 \pm 0.7$ \\
$(N=3)$ & $(n=15)$ & $(n=15)$ & $(n=15)$ & $(n=6)$ & $(n=14)$
\end{tabular}

Excitability parameters were measured with intracellular microelectrodes in $n$ fibers from $N$ mice (in brackets). The parameters were the rheobase current $\left(I_{\mathrm{th}}\right)$, the latency of first action potential (Lat), the amplitude of first action potential (APA), the ratio between $I_{\text {th }}$ and the current threshold for eliciting more than two action potentials $\left(I_{2}\right)$, the maximum number of elicitable action potentials ( $N$ spikes). Statistical analysis was performed with unpaired Student's $t$-test versus control EDL ((a) indicates at least $P<0.05)$ and Sol muscle ((b) for at least $P<0.05)$. 

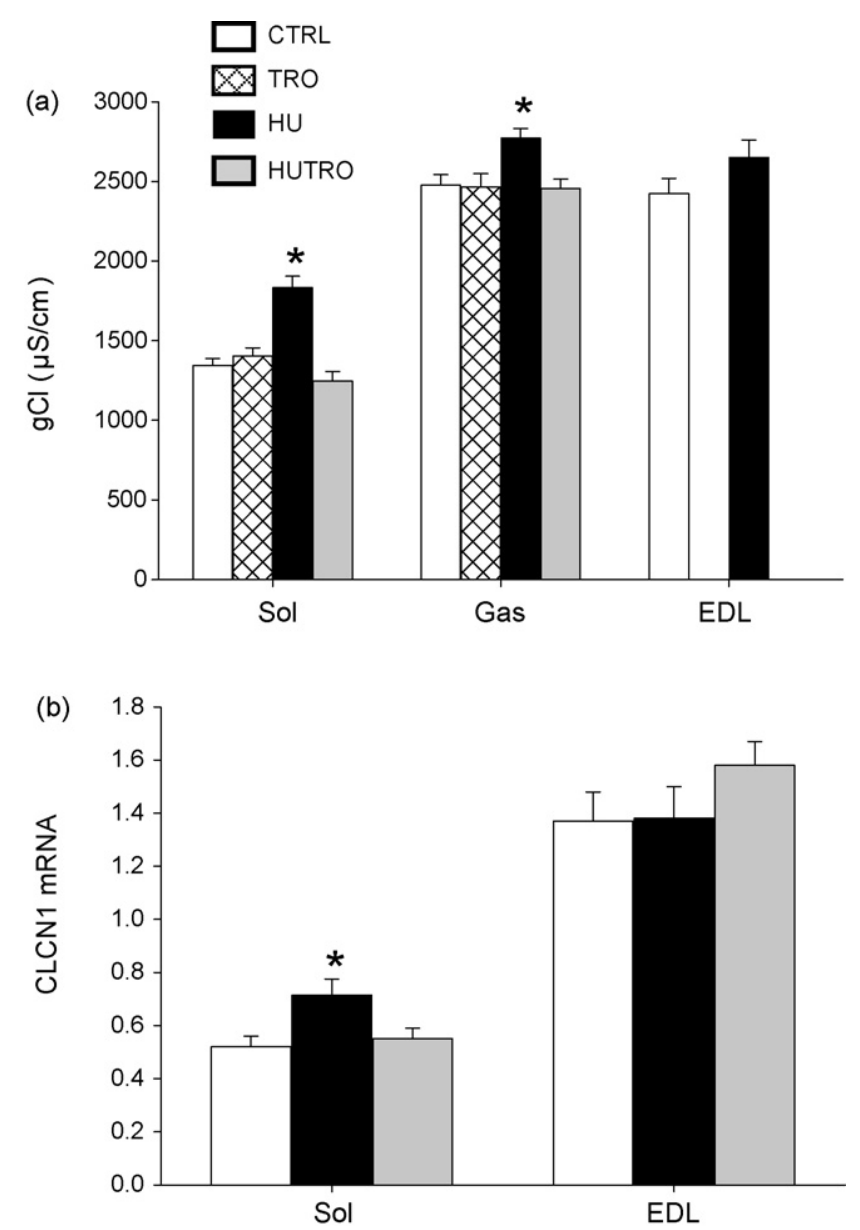

Fig. 4. Effects of HU and trolox treatment on the sarcolemma chloride conductance at rest $(\mathrm{gCl})$ and chloride $\mathrm{ClC}-1$ channel mRNA levels in mouse Sol, Gas, and EDL muscles. (a) Each bar represents the mean value $\pm \mathrm{SEM}$ of $\mathrm{gCl}$ measured in at least 27 fibers from at least 3 control (CTRL), trolox-treated mice (TRO), hindlimb-unloaded (HU), or trolox-treated HU (HUTRO) mice. For Sol and Gas muscles, statistical analysis was performed using ANOVA followed by Bonferroni's $t$-test. Significant difference (at least $P<0.005$ ) was found only between $\mathrm{HU}$ and each of the three other conditions. Regarding EDL muscle, effect of trolox treatment on $\mathrm{gCl}$ was not determined, because no significant difference was found between CTRL and HU using unpaired Student's $t$-test. (b) Levels of CLCN1 mRNA, which encodes the chloride ClC1 channel, were determined using quantitative real time PCR. Each bar represents the mean value \pm SEM of CIC- 1 mRNA normalized with respect of the housekeeping HPRT1 gene measured in Sol and EDL muscles of 4 CTRL, 3 HU, and 3 HUTRO mice. Statistical analysis was performed using ANOVA followed by Bonferroni's $t$-test. Significant difference $(P<0.05)$ was found only in Sol muscle between $\mathrm{HU}$ and each of the two other conditions.

ship, the fit of which allows the calculation of the Rheobase $R$ and of the time constant $t_{R}$ to reach $R$ (Table 2). As in the rat, Sol muscle fibers needed significantly less depolarization to contract with respect to the fast-twitch EDL muscle fibers. Little or no difference was found in the MT between fibers of the fast-twitch Gas and EDL muscle fibers. After 14 days of HU, there was no change in the MT of both fast-twitch muscles. In contrast, the Sol muscle MT was shifted toward less negative voltages after HU, in accordance with the shift of Sol muscle toward a faster phenotype. Trolox treatment of HU mice had little effect on the MT of Sol muscle, which remained significantly less negative with respect to CTRL Sol muscle.

\subsection{Effects of HU and trolox on the resting cytosolic calcium concentration (restCa)}

In rats, the cytosolic calcium concentration at rest depends on the muscle phenotype, being about two-fold in the slow-twitch Sol

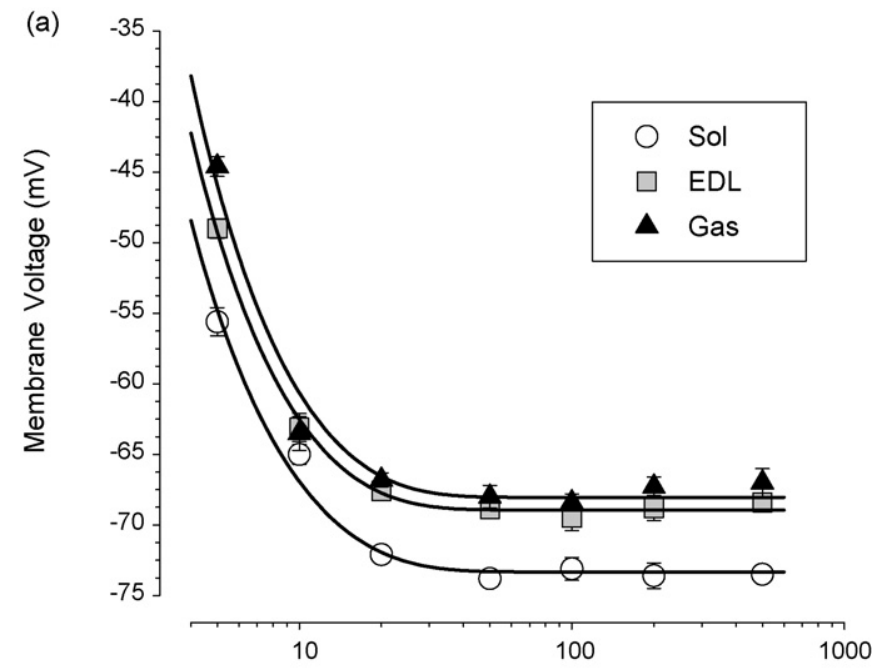

Pulse Duration (ms)

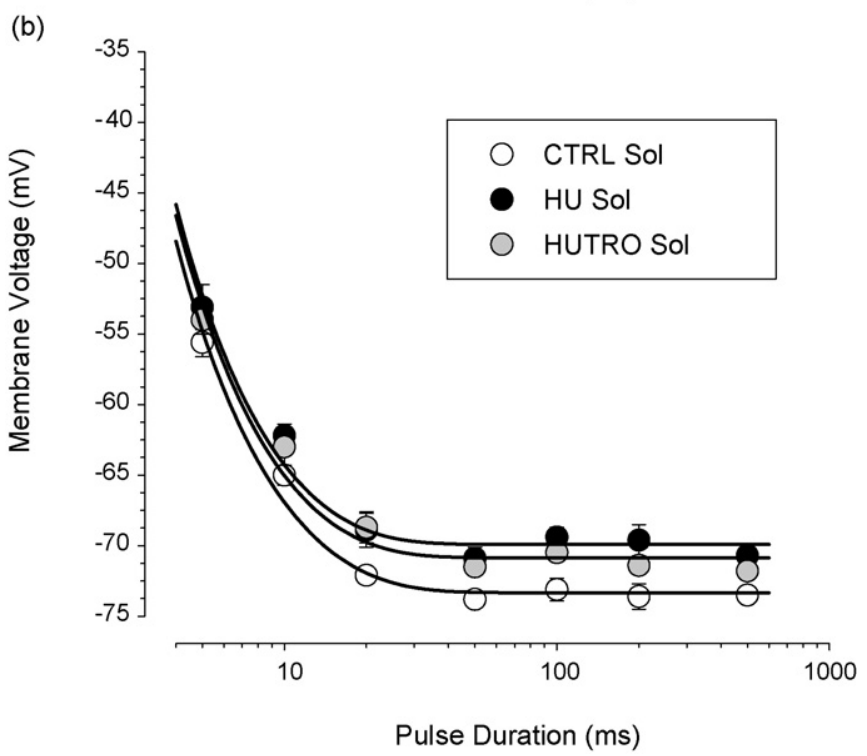

Fig. 5. Effects of HU and trolox treatment on the mechanical threshold (MT) in mouse Sol, Gas, and EDL muscles. The threshold voltage/duration relationships were obtained by plotting the values of threshold potentials $(V)$ needed to obtain myofiber contraction as a function of the voltage pulse duration $(t)$. Each point represents the mean value \pm S.E.M. of 5-29 fibers from 2 to 4 mice. In (a) are shown the relationships for Sol, EDL, and Gas muscle fibers from CTRL mice. In (b) are shown the relationships for Sol muscle fibers from CTRL, HU, and HUTRO mice. The curves fitting the experimental points were obtained using the equation $V(t)=\left[-90-R \cdot \exp \left(-t / \tau_{R}\right)\right] /\left[1-\exp \left(-t / \tau_{R}\right)\right]$, where $R$ is the Rheobase $(\mathrm{mV})$ and $\tau_{R}$ is the time constant (ms) needed to reach $R$. Fit values for $R$ and $\tau_{R}$ are given in Table 1 along with the mean standard error of the fit. The relationships obtained in EDL and Gas muscle fibers of HU mice are not shown because there was no significant difference with CTRL mice (see Table 1 ).

muscle compared to the fast-twitch EDL muscle [7]. A similar difference was also found in mice between Sol muscle fibers and fibers from the fast-twitch flexor digitorum brevis (FDB) or TA muscle fibers $[16,23]$. Our results confirm such a difference (Fig. 6). In CTRL mice, the restCa values of soleus muscle fibers was $\sim 2$-fold that measured in EDL and Gas muscle fibers. After HU, no change in restCa was observed in the fast-twitch muscles, whereas the restCa of Sol muscle fibers was significantly reduced by $\sim 25 \%$, thereby reaching an intermediate value between those of CTRL Sol muscle fibers and fast-twitch muscle fibers. With respect to HU mice, the treatment with trolox produced a trend toward a reduction of restCa in all the 
Table 2

Effects of hindlimb unloading (HU) and concomitant trolox treatment (HUTRO) on the mechanical threshold (MT) parameters.

\begin{tabular}{llrll}
\hline Muscle & Condition & \multicolumn{1}{c}{$d / N$} & Rheobase $(R, \mathrm{mV})$ & Time constant $\left(t_{R}, \mathrm{mV}\right)$ \\
\hline \multirow{2}{*}{ Sol } & CTRL & $123 / 3$ & $-73.3 \pm 0.5$ & $-7.8 \pm 0.4$ \\
& HU & $89 / 3$ & $-69.9 \pm 0.5^{*}$ & $-6.6 \pm 0.4$ \\
& HUTRO & $137 / 4$ & $-70.9 \pm 0.6^{*}$ & $-6.9 \pm 0.5$ \\
\multirow{2}{*}{ Gas } & CTRL & $78 / 2$ & $-68.0 \pm 0.7^{*} \dagger \ddagger$ & $-7.3 \pm 0.5$ \\
& HU & $104 / 3$ & $-67.1 \pm 0.9^{*} \dagger \ddagger$ & $-7.3 \pm 0.2$ \\
EDL & CTRL & $68 / 3$ & $-68.9 \pm 0.1^{*} \ddagger$ & $-6.9 \pm 0.2$ \\
& HU & $54 / 3$ & $-68.4 \pm 0.4^{*} \ddagger$ & $-6.9 \pm 0.2$ \\
\hline
\end{tabular}

Values of Rheobase and time constant are given as means \pm S.E.M. of the fit of the voltage threshold-duration relationship shown in Fig. 4. The $d$ value indicates the number of voltage threshold determinations performed in $N$ animals/muscles. Statistical analysis was performed using ANOVA followed by Bonferroni's $t$-test. Significant differences (at least $P<0.05$ ) are indicated by $\left({ }^{*}\right)$ versus control Sol, $(\dagger)$ versus HU Sol, and ( $\ddagger$ ) versus HUTRO Sol.

three muscles, although statistical significance was reached only for Gas muscle fibers.

\subsection{Effects of $\mathrm{HU}$ and trolox on muscle lipid peroxidation}

Oxidative stress has been shown to occur in Sol muscles of HU rats and mice [24-26]. We verified that this also occurred in mice after 14 days of $\mathrm{HU}$, by measuring an indicator of oxidative stress, that is the malondialdehyde (MDA), in Sol muscles. We also extended the study to three other hindlimb muscles with various degrees of HU-induced atrophy (Gas, TA, and EDL). The results are shown in Fig. 7. In CTRL mice, the MDA levels were higher in Sol muscle compared to the three fast-twitch muscles, which may be related to the oxidative metabolism of the slow muscle compared to the metabolism mainly glycolytic of the fast muscles. Compared to CTRL mice, the MDA level was significantly higher in Sol muscle after HU. A trend toward an increase in MDA levels was also observed in the other three muscles, being more pronounced in Gas, then in TA, and finally in EDL. Interestingly, although the differences in MDA levels with respect to CTRL muscles did not reach statistical significance for Gas, TA and EDL, a statistically significant linear correlation was found between the percent decrease in muscle-to-body weight ratio and the percent increase in MDA

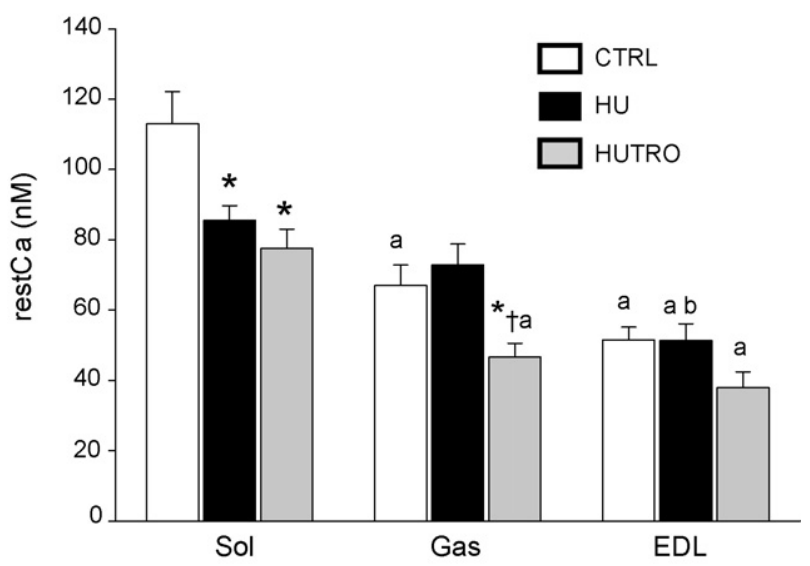

Fig. 6. Effects of HU and trolox treatment on the resting cytosolic calcium concentration (restCa) in mouse Sol, Gas, and EDL muscles. The restCa was determined by Fura-2 fluorescence imaging in single myofibers mechanically dissociated from Sol, Gas, and EDL muscles of CTRL, HU, and HUTRO mice. Each bar represents the mean \pm S.E.M. from 9 to 76 fibers of 2-9 mice. Statistical analysis was performed using ANOVA followed by Bonferroni's $t$-test to compare experimental conditions within each muscle type [at least $P<0.01$ versus CTRL $\left({ }^{*}\right)$ or versus $\mathrm{HU}(\dagger)$ ] or muscle types for each experimental condition [at least $P<0.05$ versus Sol (a) or versus Gas (b)]. levels (Fig. 7d). Importantly, the MDA levels remained similar to control level in HU mice receiving trolox, confirming that the drug exerted the expected antioxidant effect.

\section{Discussion}

Muscle fibers respond to a reduced activity by a partial slowto-fast transition of their phenotypic properties, which may in turn contribute to functional impairment, as it occurs after $\beta 2$ adrenoceptor stimulation [2]. Oxidative stress has been measured in various models of muscle disuse $[10,11]$. Antioxidant therapy has been tested as a possible countermeasure, with more or less success [12,26-30]. Most of these studies focalized more on the disuse-associated atrophic process than on the disuse-associated phenotypic transition. In the present study, we found that a strong antioxidant molecule, trolox, was unable to protect disused muscle from atrophy in the HU mouse, but partially prevented fiber type transition and changes in phenotype-dependent functional parameters.

The hindlimb-unloading model has long been used to study the effects of inactivity on skeletal muscles, especially using rats [31]. The hallmarks of this model are the atrophy and the phenotypic transition of postural muscles such as the slow-twitch soleus muscle. Using the HU rat, we also found profound changes in ion channel expression/function and $\mathrm{Ca}^{2+}$ homeostasis, which likely contribute to functional alteration as well as in modulation of gene transcription [4,6-8]. In the present study, 14 days of HU induced a dramatic atrophy of Sol muscles of mice with a $\sim 25 \%$ reduction of the muscleto-body weight ratio, which is quite similar to that observed in the HU rat. The EDL muscle, which had an almost homogenous MHC-2B content, showed no atrophy in the HU mouse, in accord with findings in HU rats. However, significant atrophy of Gas and TA muscles, which also are quite homogeneous for MHC-2B, was observed in the HU mouse. This observation suggests that differential atrophy of Sol, Gas, TA and EDL muscles may arise more from their specific role in postural maintenance than from their phenotype.

Effects of HU on MHC distribution in mouse Sol muscle consisted in a reduction of MHC-2A and an increase in MHC-2X and MHC-2B, with no change in MHC-1. Thus, in the mouse, the fast-toslow ratio of $\mathrm{MHC}$ isoforms did not change, but a transition within the fast fiber population occurred in the direction $2 \mathrm{~A}>2 \mathrm{X}>2 \mathrm{~B}$. This is lightly different from the HU rat, where expression of MHC-2A increased with MHC-2X, while little or no MHC-2B were detected $[4,32,33]$. Such a difference may rely on the different basal conditions in rats and mice; in the rat, the Sol muscle expresses mainly the slow MHC-1 isoform to support body weight upon 1G gravity. Comparatively, in similar conditions, the mouse Sol muscle already expressed a significant proportion of fast MHC isoforms, probably in relation to the smaller body size and higher level of activity of the small rodent. After unloading, the Sol muscles of both species adapt by shifting MHC isoform expression toward a faster phenotype, but starting from this different basal condition (Fig. 8). In the Sol muscle of HU rats, we previously measured changes in functional parameters such as the $\mathrm{gCl}$, the $\mathrm{MT}$, and the restCa, which we interpreted as a consequence of the slow-to-fast gene program transition [4,7]. In CTRL mice, the $\mathrm{gCl}$ is $\sim 45 \%$ lower, the MT rheobase $\sim 5 \mathrm{mV}$ more negative, and the restCa $\sim 25 \%$ higher, in the Sol muscle compared to the EDL or Gas muscles. Thus, although the Sol muscle of CTRL mice contains $60 \%$ of fast MHC isoforms, the muscle type-dependent differences in $\mathrm{gCl}$, MT rheobase, and restCa, recapitulated those observed in the HU rat. Compared to control mice, the $\mathrm{gCl}$ increased by $\sim 50 \%$, the Rheobase became less negative, and the restCa decreased in the Sol muscle of HU mice, in accord with the shift toward a faster phenotype and the tonic-to-phasic shift of Sol muscle activity [34]. 

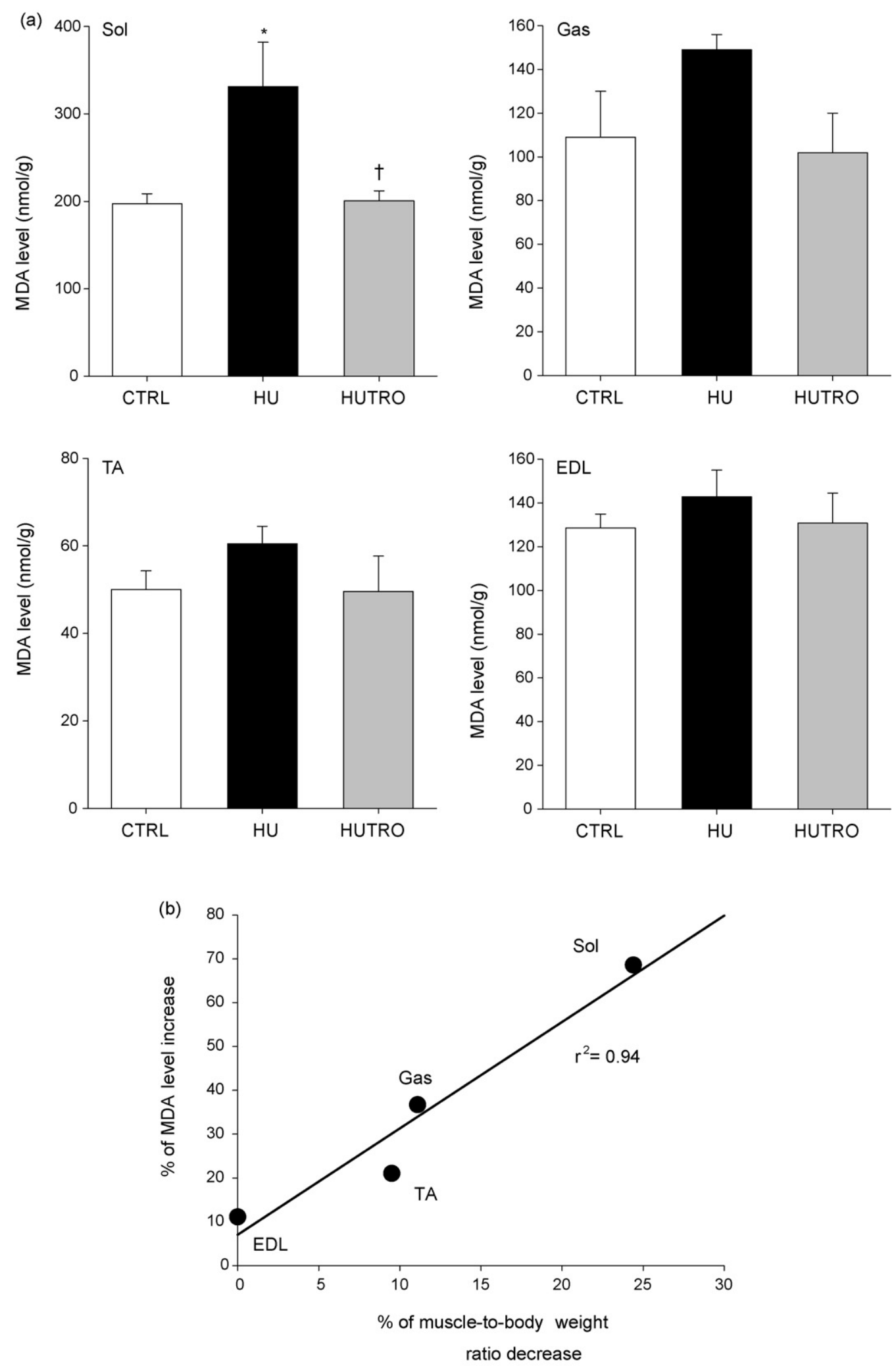

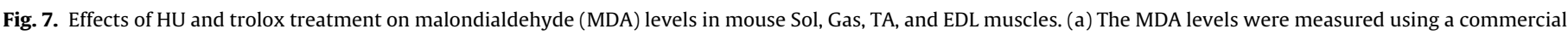

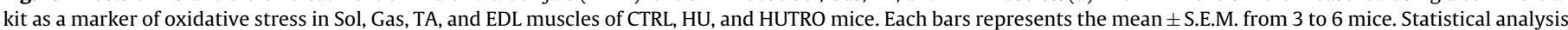

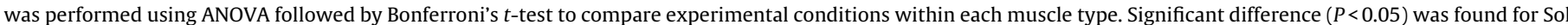

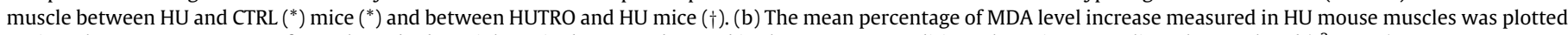
against the mean percentage of muscle-to-body weight ratio decrease observed in the same HU condition. The points were linearly correlated $\left(r^{2}=0.94\right)$. 


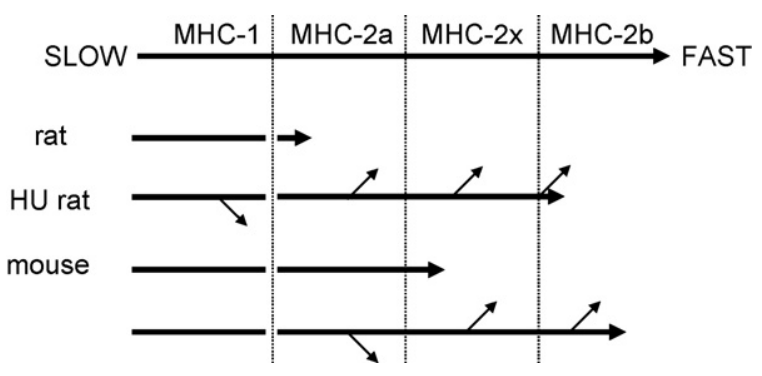

Fig. 8. Schematic diagram of HU-induced MHC transition in rat and mouse. In Sol muscles of CTRL rats, the myosin heavy chain isoform composition consists of $90 \%$ slow MHC-1 isoform and 10\% of fast MHC-2a isoform. After HU, a slow-to-fast transition takes place, with a decrease of MHC-1 proportion (downward arrow), an increase of MHC-2a content (upward arrow) and appearance of MHC-2x isoform (upward arrow) [4]. Some studies also reported appearance of MHC-2b isoform (Stevens et al. [32,33]). In CTRL mice, the Sol muscle is a mixed muscle containing $40 \%$ MHC-1, 50\% MHC-2a, and 10\% MHC-2x. As in the rat, a phenotype transition occurs after HU with a decrease of MHC-2a (downward arrow), an increase in MHC-2x and MHC-2b isoform (upward arrow) (current study).

In agreement with previous studies performed in rats [25,26], we found that HU induces oxidative stress in mouse Sol muscle, as indicated by the enhanced lipoperoxidation. We found also evidences for oxidative stress occurring in the Gas muscle of HU mice, in accord with recent observations in the HU rat [35]. However, to our knowledge, this is the first study that compares signs of oxidative stress in various slow and fast-twitch muscles of HU rodents. The good correlation between the percentage of decrease in muscle-to-body weight ratio and the percentage of increase in MDA levels (Fig. 7d) suggests a possible relationship between atrophy and oxidative stress. However, trolox treatment was unable to prevent the drop of muscle-to-body weight ratio induced by $\mathrm{HU}$ although it fully prevented lipid peroxidation, thereby suggesting that, in the HU mouse, oxidative stress is unlikely to play major role in triggering muscle atrophy.

Two antioxidants, vitamin E and Bowman-Birk inhibitor concentrate, have been previously shown to blunt atrophy in HU rodents $[24,26]$. It was however proposed that these compounds may have exerted some anti-atrophic effects independently of their antioxidant capacity [26]. Indeed, in accord with our results, two other studies have found no beneficial effect of antioxidant regimens against HU-induced atrophy $[28,30]$. Because antioxidant regimens, including trolox, have been shown to counteract, at least partially, muscle wasting in other models of reduced activity, such as limb immobilization or controlled mechanical ventilation $[12,27,29]$, this suggests that not all the models of muscle wasting may present the same molecular mechanisms responsible for atrophy. Since in vitro studies clearly indicate that oxidative stress can trigger atrophy [36,37], our results suggest that the level of oxidative stress occurring during HU may not be sufficient to contribute significantly to atrophy in this specific model. It appears more probable that oxidative stress is a product of the atrophic process or that both atrophy and oxidative stress may be induced quite independently by HU. For instance, increased production of NO through dislocation of neuronal NOS was recently shown to occur during HU, being involved in the regulation of Foxo transcription factors that are central players in atrophy [38]. It is however noteworthy that, besides its various physiological functions, NO may also constitute an important source of oxidative stress.

Besides the lack of effect of trolox on muscle atrophy, one major result of this study is the partial inhibition of MHC isoforms redistribution in Sol muscle as well as the prevention of $\mathrm{gCl}$ increase in Sol and Gas muscles by trolox treatment. This suggests that, as in the rat, the phenotypic transition and the $\mathrm{gCl}$ increase after $\mathrm{HU}$ may be closely related. Remarkably, the reduction of the $\mathrm{gCl}$ in fast-twitch muscles of transgenic mice or after pharmacologic block induces a fast-to-slow transition of the phenotype $[39,40]$. We can hypothesize that oxidative stress produced by HU would contribute to increase the $\mathrm{gCl}$, which in turn would stimulate the slow-to-fast shift in muscle phenotype. Thus a possible molecular mechanism for the increased $\mathrm{gCl}$ by oxidative stress may be the loss of nucleotide-mediated inhibition of the oxidized $\mathrm{ClC}-1$ channel [41]. Real time PCR experiments however suggest that oxidative stress may also modulate transcription of $\mathrm{ClC}-1$ channels. Importantly, the increase of $\mathrm{gCl}$ in $\mathrm{HU}$ mouse Sol muscle was associated with alteration of muscle excitability, as in the HU rat [8]. The control of $\mathrm{gCl}$ by trolox thus prevented such functional alteration. It is also worth noting that long exposure to oxidants can impair muscle contractile performance and that, although the antioxidant allopurinol did not prevent Sol muscle atrophy in HU mice, the drug was able to mitigate muscle contractile dysfunction [30,42]. On an other hand, the lack of prevention by trolox not only of muscle atrophy, but also of the MT rheobase shift and the restCa drop in HU Sol muscle indicates that trolox treatment is not sufficient to fully counteract muscle dysfunction and questions the relation between modulation of MHC expression and the restCa [7].

The data presented here strongly suggest that atrophy and muscle phenotype transition are two independent processes occurring during disuse. The prevention or correction of atrophy is required to maintain normal muscle mass, allowing muscles to develop enough force upon return to normal use. On the other hand, the phenotype allows each individual muscle to respond adequately to specific demands, in term of excitability, contraction kinetics, and resistance to fatigue. Thus muscle phenotype maintenance is necessary as well. Both atrophy and phenotype transition are very slowly and probably not completely reversible, and no efficient countermeasure is available today. Our study suggests that antioxidants may be important to prevent phenotype transition, while other studies are needed to individuate useful anti-atrophic drugs.

In conclusion, $\mathrm{HU}$ induces atrophy and a shift toward faster/ glycolytic phenotype of mouse Sol muscle. The different degrees of atrophy found in fast-twitch muscles of HU mice likely reflect the specific role of each muscle in supporting loading. Oxidative stress was increased during $\mathrm{HU}$ in parallel to muscle wasting, but antioxidant treatment was not able to prevent atrophy. In contrast, trolox was able to prevent partially the phenotypic change of Sol muscle. A number of experimental data suggest that forced fastto-slow conversion may be considered as a therapeutic strategy to protect muscle in various physiopathological conditions $[3,43]$. Thus the attenuation of Sol muscle phenotypic transition by trolox calls attention on the possible benefits of antioxidant countermeasure in protecting muscle function. A promising approach might be to develop a combined therapy associating antioxidants to reduce phenotype transition with other drugs able to limit atrophy.

\section{Acknowledgement}

This study was supported by research grants from the Italian Space Agency to D.C.C. and R.B. (project OSMA “Osteoporosis and Muscle Atrophy").

\section{References}

[1] Talmadge RJ. Myosin heavy chain isoform expression following reduced neuromuscular activity: potential regulatory mechanisms. Muscle Nerve 2000;23:661-79.

[2] Baker DJ, Constantin-Teodosiu D, Jones SW, Timmons JA, Greenhaff PL. Chronic treatment with the $\beta 2$-adrenoceptor agonist prodrug BRL-47672 impairs rat skeletal muscle function by inducing a comprehensive shift to a faster muscle phenotype. J Pharmacol Exp Ther 2006;319:439-46.

[3] Schiaffino S, Sandri M, Murgia M. Activity-dependent signaling pathways controlling muscle diversity and plasticity. Physiology (Bethesda) 2007;22:269-78. 
[4] Pierno S, Desaphy J-F, Liantonio A, De Bellis M, Bianco G, De Luca A, et al. Changes of chloride ion channel conductance of slow-to-fast fibre type transition during unloading-induced muscle disuse. Brain 2002;125:1510-21.

[5] Desaphy J-F, Pierno S, Liantonio A, De Luca A, Didonna MP, Frigeri A, et al. Recovery of the soleus muscle after short- and long-term disuse induced by hindlimb unloading: effects on the electrical properties and myosin heavy chain profile. Neurobiol Dis 2005;18:356-65.

[6] Desaphy J-F, Pierno S, Léoty C, George Jr AL, De Luca A, Conte Camerino D. Skeletal muscle disuse induces fibre type-dependent enhancement of $\mathrm{Na}^{+}$channel expression. Brain 2001;124:1100-13.

[7] Fraysse B, Desaphy J-F, Pierno S, De Luca A, Liantonio A, Mitolo CI, et al. Decrease in resting calcium and calcium entry associated with slow-to-fast transition in unloaded rat soleus muscle. FASEB J 2003;17:1916-8.

[8] Pierno S, Desaphy J-F, Liantonio A, De Luca A, Zarilli A, Mastrofrancesco L, et al. Disuse of rat muscle in vivo reduces protein kinase $C$ activity controlling the sarcolemma chloride conductance. J Physiol 2007;584:983-95.

[9] Tricarico D, Mele A, Conte Camerino D. Phenotypic-dependent functional and pharmacological properties of BK channels in skeletal muscle: effects of microgravity. Neurobiol Dis 2005;20:296-302.

[10] Moylan JS, Reid MB. Oxidative stress, chronic disease, and muscle wasting. Muscle Nerve 2007;35:411-29.

[11] Powers SK, Kavazis AN, McClung JM. Oxidative stress and disuse muscle atrophy. J Appl Physiol 2007;102:2389-97.

[12] McClung JM, Kavazis AN, Whidden KC, DeRuisseau KC, Falk DJ, Criswell DS, et al. Antioxidant administration attenuates mechanical ventilation-induced rat diaphragm muscle atrophy independent of protein kinase B (PKB-Akt) signaling. J Physiol 2007;585:203-15.

[13] Sagach VF, Scrosati M, Fielding J, Rossoni G, Galli C, Visioli F. The water-soluble vitamin $\mathrm{E}$ analogue trolox protects against ischaemia/reperfusion damage in vitro and ex vivo. A comparison with vitamin E. Pharmacol Res 2002;45: 435-9.

[14] Bryant SH, Conte Camerino D. Chloride channel regulation in the skeletal muscle of normal and myotonic goats. Pflügers Arch 1991;417:605-10.

[15] Grynkiewicz G, Poenie M, Tsien RY. A new generation of $\mathrm{Ca}^{2+}$ indicators with greatly improved fluorescence properties. J Biol Chem 1985;260:3440-50.

[16] Gailly P, Boland B, Himpens B, Casteels R, Gillis JM. Critical evaluation of cytosolic calcium determination in resting muscle fibres from normal and dystrophic (mdx) mice. Cell Calcium 1993;14:473-83.

[17] Talmadge RJ, Roy RR. Electrophoretic separation of rat skeletal muscle myosin heavy-chain isoforms. J Appl Physiol 1993;75:2337-40.

[18] Laemmli UK. Cleavage of structural proteins during the assembly of the head of bacteriophage T4. Nature 1970;227:680-5.

[19] Lowry OH, Rosebrough NJ, Farr AL, Randall RJ. Protein measurement with the Folin phenol reagent. J Biol Chem 1951;193:265-75.

[20] Pellegrino MA, Canepari M, Rossi R, D’Antona G, Reggiani C, Bottinelli R. Orthologous myosin isoforms and scaling of shortening velocity with body size in mouse, rat, rabbit and human muscles. J Physiol 2003;546:677-89.

[21] Stelzer JE, Widrick JJ. Effect of hindlimb suspension on the functional properties of slow and fast soleus fibers from three strains of mice. J Appl Physiol 2003;95:2425-33.

[22] Klocke R, Steinmeyer K, Jentsch TJ, Jockusch H. Role of innervation, excitability, and myogenic factors in the expression of the muscular chloride channel ClC-1. J Biol Chem 1994;269:27635-9.

[23] Perez CF, Lopez JR, Allen PD. Expression levels of RyR1 and RyR3 control resting free $\mathrm{Ca}^{2+}$ in skeletal muscle. Am J Physiol Cell Physiol 2005;288:640-9.
[24] Arbogast S, Smith J, Matuszczak Y, Hardin BJ, Moylan JS, Smith JD, et al. Bowman-Birk inhibitor concentrate prevents atrophy, weakness, and oxidative stress in soleus muscle of hindlimb-unloaded mice. J Appl Physiol 2007; 102:956-64.

[25] Lawler JM, Song W, Demaree SR. Hindlimb unloading increases oxidative stress and disrupts antioxidant capacity in skeletal muscle. Free Radic Biol Med 2003;35:9-16.

[26] Servais S, Letexier D, Favier R, Duchamp C, Desplanches D. Prevention of unloading-induced atrophy by vitamin E supplementation: links between oxidative stress and soleus muscle proteolysis? Free Radic Biol Med 2007;42:627-35

[27] Appell HJ, Duarte JAR, Soares JMC. Supplementation of vitamin E may attenuate skeletal muscle immobilization atrophy. Int J Sports Med 1997;18:157-60.

[28] Koesterer TJ, Dodd SL, Powers S. Increased antioxidant capacity does not attenuate muscle atrophy caused by unweighting. J Appl Physiol 2002;93: 1959-65.

[29] Kondo H, Miura M, Nakagaki I, Sasaki S, Itokawa Y. Trace element movement and oxidative stress in skeletal muscle atrophied by immobilization. Am J Physiol Endocrinol Metab 1992;262:E583-90.

[30] Matuszczak Y, Arbogast S, Reid MB. Allopurinol mitigates muscle contractile dysfunction caused by hindlimb unloading in mice. Aviat Space Environ Med 2004;75:581-8.

[31] Morey-Holton E, Globus RK, Kaplansky A, Durnova G. The hindlimb unloading rat model: literature overview, technique update and comparison with space flight data. Adv Space Biol Med 2005;10:7-40.

[32] Stevens L, Sultan KR, Peuker H, Gohlsch B, Mounier Y, Pette D. Time-dependent changes in myosin heavy chain mRNA and protein isoforms in unloading soleus muscle of rat. Am J Physiol Cell Physiol 1999;277:C1044-9.

[33] Stevens L, Gohlsch B, Mounier Y, Pette D. Changes in myosin heavy chain mRNA and protein isoforms in single fibers of unloaded rat soleus muscle. FEBS Lett 1999;463:15-8.

[34] De-Doncker L, Kasri M, Piquet F, Falempin M. Physiologically adaptive changes of the L5 afferent neurogram and of the rat soleus EMG activity during 14 days of hindlimb unloading and recovery. J Exp Biol 2005;208:4585-92.

[35] Siu PM, Pistilli EE, Always SE. Age-dependent increase in oxidative stress in gastrocnemius muscle with unloading. J Appl Physiol 2008;105:1695-705.

[36] Li YP, Chen Y, Li AS, Reid MB. Hydrogen peroxide stimulates ubiquitinconjugating activity and expression of genes for specific E2 and E3 proteins in skeletal muscle myotubes. Am J Physiol Cell Physiol 2003;285:C806-12.

[37] McClung JM, Judge AR, Talbert EE, Powers SK. Calpain-1 is required for hydrogen peroxide-induced myotube atrophy. Am J Physiol Cell Physiol 2009;296:C363-71.

[38] Suzuki N, Motohashi N, Uezumi A, Fukada S-I, Yoshimura T, Itoyama Y, et al. NO production results in suspension-induced muscle atrophy through dislocation of neuronal NOS. J Clin Invest 2007;117:2468-76.

[39] Salviati G, Biasia E, Betto R, Danieli-Betto D. Fast to slow transition induced by experimental myotonia in rat EDL muscle. Pflugers Arch 1986;406:266-72.

[40] Wu H, Olson EN. Activation of MEF2 transcription factor in skeletal muscles from myotonic mice. J Clin Invest 2002;109:1327-33.

[41] Zhang X-D, Tseng P-Y, Chen T-Y. ATP inhibition of ClC-1 is controlled by oxidation and reduction. J Gen Physiol 2008;132:421-8.

[42] Lamb GD, Posterino GS. Effects of oxidation and reduction on contractile function in skeletal muscle fibres of the rat. J Physiol 2003;546:149-63.

[43] Hilber K. Skeletal myocyte plasticity: basis for improved therapeutic potential. Curr Opin Pharmacol 2008;8:327-32. 